\title{
COMPOSITIONS OF CONTINUOUS FUNCTIONS AND CONNECTED FUNCTIONS
}

\author{
KENNETH R. KELLUM AND HARVEY ROSEN
}

(Communicated by R. Daniel Mauldin)

\begin{abstract}
Suppose $f: X \rightarrow Y$ is continuous and onto and $g: Y \rightarrow Z$ is such that $g \circ f: X \rightarrow Z$ has a property we are interested in. For which properties of functions can we infer that $g$ has the same property? Properties for which we can infer this include continuity and the Darboux property. Properties for which we cannot include almost continuity.
\end{abstract}

In calculus, we encounter the result that the composition $g \circ f$ of two continuous functions $f: I \rightarrow I$ and $g: I \rightarrow I$ is continuous, where $I=[0,1]$. This brings to mind the following question: If $f: X \rightarrow Y$ is continuous and onto, what property should $g \circ f$ have in order for $g: Y \rightarrow Z$ to possess the same property? For example, if $f$ and $g \circ f$ are continuous, will $g$ be continuous? The reason we suppose $f$ is onto $Y$ is that $g \circ f$ would be continuous when $g$ is continuous on $f(X)$, but not on $X \backslash f(X)$. Of course, there are many properties of functions for which this question is of interest. We consider only a few of our favorites here.

If $h$ is any function, we let $\operatorname{Gr}(h)$ denote the graph of $h$. Suppose $f: X \rightarrow$ $Y$ is continuous and onto and $g: Y \rightarrow Z$. The height-preserving function $H: X \times Z \rightarrow Y \times Z$ defined by $H(x, y)=(f(x), y)$ is continuous. Now,

$$
\begin{aligned}
H(\operatorname{Gr}(g \circ f)) & =H(\{(x, g(f(x))): x \in X\}) \\
& =\{(f(x), g(f(x))): x \in X\}=\operatorname{Gr}(g)
\end{aligned}
$$

because $f$ is onto $X$. This observation immediately gives us the following two theorems, the first being an easy consequence of the closed graph theorem.

Theorem 1. If $f: X \rightarrow Y$ is continuous and onto and if $g \circ f: X \rightarrow Z$ is continuous where $X, Y$, and $Z$ are compact, then $g: Y \rightarrow Z$ is continuous.

Theorem 2. If $f: X \rightarrow Y$ is continuous and onto and if $g \circ f: X \rightarrow Z$ has a connected graph, then $g: Y \rightarrow Z$ has a connected graph.

However, if $f: X \rightarrow Y$ is not an identification, then according to Theorem 3.1 of [1], there exist a space $Z$ and a function $g: Y \rightarrow Z$ where $g$ is not continuous but $g \circ f$ is continuous.

Received by the editors October 15, 1990.

1980 Mathematics Subject Classification (1985 Revision). Primary 26A15, 54C08, 54C30.

Key words and phrases. Darboux property, connectivity function, almost continuous function. 
Let $\mathfrak{R}$ denote the set of real numbers with the usual topology.

A function is said to be a Darboux function if the image of each connected set is connected.

Theorem 3. Suppose $X$ is a metric continuum and $f: X \rightarrow I$ is continuous and onto. If $g \circ f: X \rightarrow \mathfrak{R}$ is a Darboux function, then so is $g: I \rightarrow \mathfrak{R}$.

Proof. Suppose $a<b$ and $z$ lies between $g(a)$ and $g(b)$. We show there exists a number $c$ between $a$ and $b$ such that $g(c)=z$. Let $N$ be a component of $f^{-1}(a, b)$. According to Theorem 2-16 of [7], $\operatorname{cl}\left(f^{-1}(a, b)\right) \backslash f^{-1}(a, b)$ contains a limit point of $N$. Therefore, since $f$ is continuous, $\operatorname{cl}(N)$ meets either $f^{-1}(a)$ or $f^{-1}(b)$. Let $\mathscr{C}$ denote the collection of all components $C$ of $f^{-1}(a, b)$ such that $\operatorname{cl}(C)$ meets $f^{-1}(a)$, and let $\mathscr{D}$ denote the collection of all components $D$ of $f^{-1}(a, b)$ such that $\operatorname{cl}(D)$ meets $f^{-1}(b)$.

Here is how to obtain a connected set $M$ in $f^{-1}[a, b]$ that meets both $f^{-1}(a)$ and $f^{-1}(b)$. In case there is a member $C$ of $\mathscr{C} \cap \mathscr{D}$, let $M=\operatorname{cl}(C)$. Suppose $\mathscr{C} \cap \mathscr{D}=\varnothing . \quad H=f^{-1}[0, a]$ and $K=f^{-1}[b, 1]$ are separated sets. Let $A=H \cup(\bigcup \mathscr{C})$ and $B=K \cup(\cup \mathscr{D})$. Since $X$ is connected and $X=A \cup B$, we may suppose, for argument's sake, that $B \cap \operatorname{cl}(A) \neq \varnothing$. Then there is a point $p \in B \cap \operatorname{cl}(\cup \mathscr{C})$. Let $\left\{x_{n}\right\}$ be a sequence in $\cup \mathscr{C}$ converging to $p$, and let $C_{n}$ be the member of $\mathscr{C}$ to which $x_{n}$ belongs. For each $n$, choose $y_{n} \in f^{-1}(a) \cap \operatorname{cl}\left(C_{n}\right)$. For simplicity, we may suppose $\left\{y_{n}\right\}$ already converges to a point $q$. Then $q \in f^{-1}(a)$. By Theorem 2-101 of [7], $Y=\lim \sup C_{n}$ is connected, and $p, q \in Y$. In case $p \in K$, then $Y$ meets $f^{-1}(a)$ and $f^{-1}(b)$, so choose $M=Y$. In case $p \in D_{n}$ for some $D_{n} \in \mathscr{D}$, choose $M$ to be the connected subset $Y \cup \operatorname{cl}\left(D_{n}\right)$ of $f^{-1}[a, b]$ which then meets both $f^{-1}(a)$ and $f^{-1}(b)$.

Now, $f(M)=[a, b]$. Since $g \circ f$ is a Darboux function, $(g \circ f)(M)$ is connected, and $(g \circ f)(M)=g[a, b]$. Therefore there exists a number $w \in M$ such that $g(f(w))=z$. Choosing $c=f(w)$, we have $a<c<b$ and $g(c)=z$.

A function $f: X \rightarrow Y$ is almost continuous if, whenever $D \subseteq X \times Y$ is an open set with $\operatorname{Gr}(f) \subseteq D$, then there exists a continuous function $g: X \rightarrow Y$ such that $\operatorname{Gr}(g) \subseteq D$.

Example 1. There exist $f: I \rightarrow I$ continuous and onto and $g: I \rightarrow I$ not almost continuous such that $g \circ f$ is almost continuous.

Proof. In [8] F. B. Jones and E. S. Thomas, Jr. give an example of a function from $I$ to $I$ with a connected graph which is not almost continuous. This function will be the function $g$ in our example. Let $C$ denote the Cantor middle-third set. A property of $g$ that we will use is that if $(a, b)$ is a component of $I \backslash C$, then $\left.g\right|_{[a, b]}$ is continuous and $g(a, b)=[0,1]$.

The continuous function $f$ is defined to be the identity on $C$. Also, if $(a, b)$ is a component of $I \backslash C$, then $f$ maps $(a, b)$ onto itself and has an $\mathrm{N}$ shape. More precisely, for each such $(a, b)$, choose $c$ and $d$ such that $a<c<d<b$. Define $f$ to be continuous on $[a, b]$ so that $f(a)=f(d)=a$, $f(c)=f(b)=b, f$ is increasing on $[a, c]$ and $[d, b]$ and is decreasing on $[c, d]$.

Observe that if $(a, b)$ is a component of $I \backslash C$, then $\left.g \circ f\right|_{[a, b]}$ is continuous and there exist disjoint subintervals $[p, q]$ and $[r, s]$ of $[a, b]$ such that $g(f(p))=g(f(s))=0$, and $g(f(q))=g(f(r))=1$. 
We claim that $g \circ f$ is almost continuous. Assume that it is not. From the definition of almost continuity, there exists a closed set $K$ in $I^{2}$ such that $K \cap \operatorname{Gr}(g \circ f)=\varnothing$ and if $h: I \rightarrow I$ is continuous, then $\operatorname{Gr}(h) \cap K \neq \varnothing$. We may take $K$ to be irreducible with respect to the property of intersecting all graphs of continuous functions from $I$ into $I$ (see [9]), from which it follows that the $X$-projection, $\pi_{X}(K)$, of $K$ is connected. Since $g \circ f$ is continuous on $I \backslash C$, it is clear that $\pi_{X}(K)$ cannot be contained in the closure of any component of $I \backslash C$. Thus, there is a point $z$ of $C$ in the interior of $\pi_{X}(K)$, where $z$ is not an endpoint of a component of $I \backslash C$. By the irreducibility of $K$, there exist continuous functions $i:[0, z] \rightarrow I$ and $j:[z, 1] \rightarrow I$ such that neither $\operatorname{Gr}(i)$ nor $\operatorname{Gr}(j)$ contains a point of $K$. Either $i(z)>j(z)$ or $i(z)<j(z)$. As the two cases are similar, we assume that $i(z)>j(z)$. Choose a circular open neighborhood $U$ of $(z, j(z))$ such that $U \cap K=\varnothing$. Note that $\operatorname{Gr}(i) \cap U=\varnothing$, for otherwise we could easily construct a continuous function on $I$ whose graph misses $K$. We may choose a component $(a, b)$ of $I \backslash C$ where $(a, b) \subseteq \pi_{X}(U)$ and $b<z$. There exists an interval $[r, s]$ in $(a, b)$ such that $g(f(r))=1$ and $g(f(s))=0$. We now construct the graph of a continuous function on $I$ by taking the union of parts of the graphs of four functions. Take the part of $\operatorname{Gr}(i)$ from $(0, i(0))$ to a point $(c, i(c))$ where $i(c)=g(f(c))$ and $c \in[r, s]$. Take the part of $\operatorname{Gr}(g \circ f)$ from $(c, g(f(c)))$ down to a point $(d, g(f(d))) \in U$, with $d<z$. Now take a straight line segment from $(d, g(f(d)))$ to $(z, j(z))$, together with all of $\operatorname{Gr}(j)$. Since the graph of the resulting continuous function does not meet $K$, we have a contradiction. Thus $g \circ f$ is almost continuous.

Let $f: I \rightarrow I$ be continuous, $g: I \rightarrow I$ have a connected graph, and $h: I \rightarrow$ $I$ be almost continuous. It is known that both $h \circ f$ and $f \circ h$ are almost continuous [10] and that $f \circ g$ has a connected graph [6]. We show next that $g \circ f$ has a connected graph, too. This result seems to have been overlooked. In Theorem 2.7 of [6], Hildebrand and Sanderson did show that given topological spaces $R$ and $T$, a set $S$, and functions $f: R \rightarrow S$ and $g: S \rightarrow T$, then $g \circ f$ is a connectivity function if and only if $S$ has a topology for which $f$ is a connectivity function and $g$ is continuous.

Theorem 4. If $f: \mathfrak{R} \rightarrow \mathfrak{R}$ is continuous and $g: \mathfrak{R} \rightarrow \mathfrak{R}$ has a connected graph, then $g \circ f$ has a connected graph.

Proof. According to [2], a function $g: \mathfrak{R} \rightarrow \mathfrak{R}$ with a connected graph can be characterized in terms of compact, connected subsets of the $x, y$ plane $\mathfrak{R}^{2}$ as follows: The graph of $g$ is connected if and only if whenever $D$ is a continuum in $\mathfrak{R}^{2}$ which contains points above and below the graph of $g$, then $D$ meets the graph of $g$. With this in mind, we let $M$ be a compact, connected subset of $\mathfrak{R}^{2}$ which contains points above and below $\operatorname{Gr}(g \circ f)$. That is, there exist points $\left(a, z_{1}\right),\left(b, z_{2}\right) \in M$ such that $z_{1}>g(f(a))$ and $z_{2}<g(f(b))$. (It may happen that $a=b$ if, for instance, $M$ is a circle whose interior contains the point $(a, g(a))$.) Since the function $H: \mathfrak{R}^{2} \rightarrow \mathfrak{R}^{2}$ defined by $H(x, y)=(f(x), y)$ is continuous, $H(M)$ is compact and connected. Let $D$ denote $H(M)$. The points $H\left(a, z_{1}\right)$ and $H\left(b, z_{2}\right)$ of $D$ lie, respectively, above the graph of $g$ and below the graph of $g$. As a result, $D$ intersects the graph of $g$. Let $(c, g(c)) \in D$. So $(c, g(c))=\left(f\left(x_{0}\right), y_{0}\right)$ for some point $\left(x_{0}, y_{0}\right) \in M$. But $y_{0}=g(c)=g\left(f\left(x_{0}\right)\right)$, which implies $\left(x_{0}, y_{0}\right) \in \operatorname{Gr}(g \circ f)$, 
too. This shows that $M$ meets the graph of $g \circ f$, and, consequently, $g \circ f$ has a connected graph.

$f: X \rightarrow Y$ is a connectivity function if $\operatorname{Gr}\left(\left.f\right|_{C}\right)$ is connected whenever $C$ is connected. A function with domain $\mathfrak{R}$ is a connectivity function if and only if its graph is connected. However, Hagan [5] and Whyburn [11] have shown that a function $f: I^{n} \rightarrow I^{m}$, where $n \geq 2$, is a connectivity function if and only if $f$ is peripherally continuous. That $f$ is peripherally continuous at $x$ means that given open neighborhoods $U$ and $V$ of $x$ and $f(x)$, respectively, there exists a neighborhood $W$ of $x$ such that $x \in W \subseteq U$ and $f($ boundary $y(W)) \subseteq V$.

It is somewhat surprising that Theorem 4 holds. Indeed Stallings [10] stated that connectivity functions do not, in general, satisfy a proposition similar to our Theorem 4 and that for this reason, the study of connectivity maps is difficult. In $[10, \S 6$, p. 261] Stallings indicated how to construct a counterexample. We give two examples below for completeness.

Example 2. Define $f: I^{2} \rightarrow I$ by $f(x, y)=x$ and $g: I \rightarrow I$ by

$$
g(x)= \begin{cases}(1+\sin (2 \pi / x)) / 2 & \text { if } 0<x \leq 1 \\ 1 & \text { if } x=0\end{cases}
$$

Now, let

$$
X=(I \times\{0\}) \cup \bigcup_{i=1}^{\infty}(\{1 / i\} \times I) \cup\{(0,1)\}
$$

and let $\hat{f}$ be the restriction of $f$ to $X$. Then $\hat{f}$ is continuous, $\operatorname{Gr}(g)$ is connected, but the point $(0,1,1)$ is an isolated point of $\operatorname{Gr}(g \circ \hat{f})$. It follows that $g \circ f$ is not a connectivity function.

Example 3. There exist a continuous function $f: I^{2} \rightarrow I^{2}$ and a peripherally continuous function $g: I^{2} \rightarrow I$ such that $g \circ f$ is not peripherally continuous.

Proof. Let $A$ be an arc in $I^{2}$ and let $f: I^{2} \rightarrow I^{2}$ shrink $A$ to a point. That is, $f$ is continuous, $f(A)$ is a point, and $f$ is a homeomorphism off $A$. Define $g: I^{2} \rightarrow I$ by

$$
g(P)= \begin{cases}(1+\sin (1 / \operatorname{dist}(P, f(A)))) / 2 & \text { if } P \neq f(A) ; \\ 1 / 2 & \text { if } P=f(A) .\end{cases}
$$

Then $g$ is peripherally continuous but $g \circ f$ is not.

Gibson and Roush [3] have proved that if $f: I^{2} \rightarrow I$ is an onto continuous function and $g \circ f$ is a connectivity function, where $g: I \rightarrow I$, then $g$ is continuous except perhaps at 0 or 1 (also see [4]). It follows from our Theorem 2 that $g$ also has a connected graph.

\section{REFERENCES}

1. J. Dugundji, Topology, Allyn and Bacon, Boston, 1966.

2. B. D. Garrett, D. Nelms, and K. R. Kellum, Characterizations of connected real functions, Jber. Deutsch. Math.-Verein. 73 (1971), 131-137.

3. R. G. Gibson and F. Roush, Connectivity functions defined on $I^{n}$, Colloq. Math. 55 (1988), 41-44. 
4. R. G. Gibson, H. Rosen, and F. Roush, Compositions and continuous restrictions of connectivity functions, Topology Proc. 13 (1988), 83-91.

5. M. R. Hagan, Equivalence of connectivity maps and peripherally continuous transformations, Proc. Amer. Math. Soc. 17 (1966), 175-177.

6. S. K. Hildebrand and D. E. Sanderson, Connectivity functions and retracts, Fund. Math. 57 (1965), 237-245.

7. J. G. Hocking and G. S. Young, Topology, Addison-Wesley, Reading, MA, 1961.

8. F. B. Jones and E. S. Thomas, Jr., Connected $G_{\delta}$-graphs, Duke Math. J. 33 (1966), 341-345.

9. K. R. Kellum, Sums and limits of almost continuous functions, Colloq. Math. 31 (1974), 125-128.

10. J. Stallings, Fixed point theorems for connectivity maps, Fund. Math. 47 (1959), 249-263.

11. G. T. Whyburn, Connectivity of peripherally continuous functions, Proc. Nat. Acad. Sci. U.S.A. 55 (1966), 1040-1041.

Department of Mathematics and Computer Science, San José State University, San José, CALIFORNIA 95192

Department of Mathematics, The University of Alabama, Tuscaloosa, Alabama 35487

E-mail address: kellum@sjsumcs.sjsu.edu 